A Study Protocol for the Australasian Oncofertility Registry: Monitoring referral patterns and the uptake, quality and complications of fertility preservation strategies in Australia and New Zealand.

Anazodo A.C ${ }^{1,2,34}$, Stern C ${ }^{5,6}$, McLachlan R.I ${ }^{7,8}$, Gerst B ${ }^{3,9}$ Agresta $F^{5}$ Cohn RJ ${ }^{1,3}$, Jayasinghe $Y^{6,10}$, Wakefield C.E. ${ }^{1,3}$, Daly $G^{3}$, Chan $D^{4}$, Gilbert $L^{3}$, Kemertzis $M^{6,10}$, Orme L. $\mathbf{M}^{6,11}$, Wand $H^{9,}$, Viney $\mathbf{R}^{12}$, Gillam $L^{13}$, Deans $\mathbf{R}^{14,15}$, Jetti $\mathbf{M}^{16}, \mathbf{W u} \mathbf{J}^{16}$, Chapman $M^{14,15}$, Ledger $\mathbf{W}^{14,15}$, Sullivan E. $\mathbf{A}^{12}$.

'School of Women's and Children's Health, Discipline of Paediatrics, UNSW Medicine, University of New South Wales, New South Wales, Australia, 2031

2Sydney Youth Cancer Service, Sydney, New South Wales, Australia, 2031.

${ }^{3}$ Kids Cancer Centre, Sydney Children's Hospital, Sydney, New South Wales, Australia 4Prince of Wales Hospital, High Street Randwick, Australia 2031.

${ }^{5}$ Melbourne IVF, Victoria, Australia

${ }^{6}$ The Royal Children's Hospital, Parkville, Victoria, Australia

${ }^{7}$ Monash IVF, Goodwood Street Richmond VIC Australia

${ }^{8}$ Andrology Australia, School of Public Health and Preventive Medicine, Monash University, Prahran, Victoria, Australia

${ }^{9}$ The Kirby Institute, Wallace Wurth Building, University of New South Wales, Kensington New South Wales, Australia 2033

${ }^{10}$ Department of Obstetrics and Gynaecology, The University of Melbourne, Royal Women's Hospital, Parkville, Victoria, Australia

${ }^{11}$ Peter MacCallum Cancer Centre, East Melbourne, Victoria, Australia

${ }^{12}$ University of Technology Sydney, Broadway, New South Wales, Australia

${ }^{13}$ Children's Bioethics Centre, Royal Children's Hospital, Melbourne School of Population and Global Health, University of Melbourne

${ }^{14}$ The Royal Hospital for Women, Sydney, New South Wales, Australia

${ }^{15}$ School of Women's and Children's Health, Discipline of Obstetrics \& Gynaecology, UNSW Medicine, University of New South Wales, New South Wales, Australia ${ }^{16}$ Salesforce Australia, Tower 2 Sussex Road, Sydney, New South Wales 


\section{Correspondence to:}

Dr Antoinette Anazodo

BSc. MBBS, MRCPCH, FRACP, MSc, Diploma Adolescent Cancer

Paediatric and Adolescent Cancer Specialist

Director of the Sydney Youth Cancer Service

Sydney Children's and Prince of Wales Hospital

Chief Investigator FUTuRE Fertility Study

High Street Randwick

Randwick

Australia

2031

Phone - +61447017444

Fax- +61293821789

Email - Antoinette.anazodo@sesiahs.health.nsw.gov.au

Website -www.futurefertility.com.au

Twitter - @ANZoncofert

\section{Abstract}

Improvements in cancer diagnosis and treatment in patients of a reproductive age, have led to significant improvements in survival rates, however a patient's fertility can be affected by both cancer and its treatment. As survival rates improve, there is an expectation by clinicians and patients that patient's reproductive potential should be considered and protected as much as possible. However, there is a lack of data about current fertility preservation uptake as well as accurate data on the acute or permanent reproductive risks of cancer treatment, complications of fertility preservation in cancer patients and the use and success of assisted reproductive technology by cancer survivors. Fertility preservation remains a major gap in acute cancer management with lifelong implications for cancer survivors.

The FUTURE Fertility research team have established the first bi-national multi-site Australasian Oncofertility Registry, which is collecting a complete oncofertility dataset from 
cancer and fertility centres in Australia and New Zealand. Outcomes from the research study will monitor referral, uptake and complications of fertility preservation, document patient's reproductive potential after treatment and collect data on the use of assisted reproductive technology following cancer treatment.

The data will be linked to other routine health and administrative datasets to allow for other research projects to be carried out. The changes in oncofertility care will be benchmarked against the Australasian Oncofertility Charter. The data will be used to develop evidencebased guidelines and resources, including development of accurate risk projections for patients' risk of infertility allowing clinicians to make recommendations for fertility preservation or assisted reproductive technology.

Australian New Zealand Clinical Trials Number - 12615000221550

\section{Running Head}

Australasian Oncofertility Registry Study

\section{Key Words}

AOFR-Australasian Oncofertility Registry, ART-Assisted Reproductive Treatment, AYAAdolescent and Young Adult (15-25 year olds), CRA-Clinical Research Assistant, FP- Fertility Preservation, FPS-Fertility Preservation Services, Fertility Specialist- reproductive endocrinologist, Andrologists, paediatric gynaecologist or paediatric endocrinologist.

\section{Background}

Approximately 9,000 patients aged 0-44 years are diagnosed annually with cancer in Australia and 1,896 patients in New Zealand. ${ }^{[1,2]}$ Advances in cancer treatment have led to significant improvements in survival rates ${ }^{[1,2]}$ and clinicians are now turning their focus to the quality of survivorship. The loss of reproductive potential is one of the most distressing adverse consequences of successful cancer treatment, and can affect the quality of life of cancer survivors and lead to psychological distress. ${ }^{[3-5]}$ 
The human ovary contains a fixed pool of primordial oocytes which declines with age, culminating in menopause at an average age of 50 years. ${ }^{[6]}$ Chemotherapy and radiotherapy may deplete the ovarian reserve and cause premature ovarian insufficiency (POI). ${ }^{[7]}$ In males, spermatogenesis is extremely vulnerable to the damaging effects of chemotherapy and radiotherapy resulting in oligo/azoospermia. ${ }^{[7-9]}$

Although many survivors will maintain their reproductive potential (the ability to have a biological child), a cancer diagnosis or its treatment may cause damage to the ovaries, uterus, testes or neuroendocrine axis resulting in temporary or more commonly later onset of permanent infertility. ${ }^{[10-12]}$ Some cancers will have a direct effect on the reproductive organs or the neuroendocrine axis because of the location of the cancer or surgical procedures in this area. Most patients will have a reduced fertility potential because of the severity of their illness or type of treatment (chemotherapy, bone marrow therapy or radiotherapy) and the dose or field of treatment. ${ }^{[13]}$ Little is known about the extent to which new combination regimens or novel agents may result in an increased risk of infertility. ${ }^{[14]}$

Predicting the risk of infertility for an individual prior to the commencement of cancer treatment is difficult, particularly in paediatric or adolescent patients, and depends on multiple factors such as their age, pubertal status, gynaecological and reproductive health history, underlying medical conditions, (including genetic or endocrine conditions), cancer type, and importantly, the nature of treatment required. ${ }^{[15,16]}$

Fertility preservation (FP) is the overarching term used for medical and surgical approaches to minimise the impact of cancer treatment on future fertility. ${ }^{[17]}$ The burden of cancer-related infertility is unknown in Australia and New Zealand but this is an emerging health problem that can have an impact on a patient's quality of life and psychological wellbeing. ${ }^{[18-21]}$ In addition, both male and female patients can have medical health problems such as fatigue, delayed puberty, hypogonadism, osteoporosis and cardiovascular disease as a result of early menopause. ${ }^{[22]}$ With the development of FP strategies (oocyte, embryo, sperm, ovarian and testicular tissue cryopreservation) and oncofertility care (fertility management for cancer 
patients $)^{[17,23]}$ an increasing number of patients of or before reproductive age are being referred for FP and may be able to plan a biological family post cancer treatment ${ }^{24]}$

\section{Fertility Preservation Options}

FP options depend on a patient's age, sex, time available prior to starting cancer treatment, whether the patient has a partner at the time, type of cancer, type of cancer treatment, and also the potential malignant involvement of gonadal tissue.

Options for female patients include embryo and oocyte cryopreservation, ovarian tissue cryopreservation and ovarian suppression with gonadotropin-releasing hormone $(\mathrm{GnRH})$ agonists. ${ }^{[17,25-29]}$ Recent improvements in ovarian stimulation, oocyte and embryo freezing and ovarian tissue grafting have improved outcomes with the use of ART following FP.

Sperm cryopreservation is routinely used and is a highly reliable and well established approach for post pubertal male patients. ${ }^{[29-31]}$ Post pubertal patients who are unable to produce sperm by masturbation either due to their immaturity, or psychological or medical factors, can have semen collected using alternative methods such as electroejaculation or testicular sperm extraction (TSE). ${ }^{[32,33]}$ Men with cancer may present with subfertility at diagnosis even though gonadal or neuroendocrine tissue may not be involved. With intracytoplasmic sperm injection (ICSI), conception is now possible for men with severe oligospermia or those with azoospermia, where testicular spermatids can be extracted.

Currently, ovarian tissue cryopreservation is the only option available to pre-pubertal females cancer patients. ${ }^{[34]}$ Clinicians are optimistic about the chance of pregnancy after re-grafting of ovarian tissue in adult patients but there is still the uncertainty surrounding the prospects for live birth after regrafting of tissue collected from a pre-pubertal girl. However such tissue samples contain many primordial follicles and should yield mature oocytes when appropriately stimulated. Techniques for FP in pre-pubertal boys are limited to testicular tissue harvesting. It is hoped that in-vitro maturation techniques will provide an option for 
paediatric patients who have undergone testicular stem cell harvesting, however these remain experimental currently and unproven.[35, 36]

\section{Barriers to FP}

Several clinician and patient barriers exist in providing oncofertility care and these barriers need to be quantified more accurately in Australia and New Zealand in order to provide a standard approach to oncofertility care. ${ }^{[37-42]}$ Referral pathways between cancer and fertility clinicians vary between states and also institutions, relying sometimes on particular relationships between centres rather than a formal referral system. Data from the Australasian Oncofertility Registry will inform government bodies about access to FP, including access for rural patients and those in lower socio demographic communities.

In New Zealand, FP strategies have been covered by District Health Boards for up to 10 years. ${ }^{[43]}$ In Australia there is some public funding towards FP but it does not cover storage of gonadal tissue. Children or adolescent and young adult patients (AYA) patients will require their gonadal tissue to be stored for long periods of time and therefore this incurs additional costs for storage and requires the patient to remember details of the procedure and location of the storage many years after a cancer diagnosis.

\section{What is currently being done nationally and internationally?}

A review ${ }^{[41,42]}$ of the literature up until December 2014 highlighted the lack of published data internationally from FP databases or registries collecting 'whole of care' oncofertility information from male and female pre and post pubertal cancer patients:

- The International Society of Fertility Preservation (ISFP) launched the Ovarian Cortex freezing registry in 2014 , which is designed to collect ovarian cortex cryopreservation data. ${ }^{[44]}$

- The Northwestern Oncofertility Consortium has an observational fertility information research study (FIRST Registry) collecting annual questionnaire data from women aged 18-44 years on the impact of cancer treatment on the reproductive health of young survivors. ${ }^{[45]}$ 
- The Human Oocyte Preservation Experience (HOPE) is a prospective multicentre, observational oocyte cryopreservation registry; however this registry has not collected data from cancer patients. ${ }^{[46,47]}$

- The FertiPROTEKT Network is the European oncofertility consortium set up in 2006 to provide expertise in oncofertility and standardised support and FP treatment recommendations for female cancer patients. 70 registered centres in Germany, Switzerland and Austria collect and report on FP data prior to cancer treatment ${ }^{[48]}$

- A number of countries produce national reports about the success of assisted reproductive technologies, however to date these reports have not included specific data on FP in cancer patients. ${ }^{[49,50]}$

The Fertility Society of Australia in Australia and New Zealand in 2013 conducted a survey on fertility preservation practice and national data collection ${ }^{[51]}$. The study had $100 \%$ response rate from the medical director or manager of each of the 80 single or group fertility practices in Australia and New Zealand. Only one questionnaire was sent to each group practice. Andrology centres were not included in the study. Seventy-eight percent of the fertility centres stated they would be interested in participating in a national FP registry. The majority of the clinicians felt that it would be impractical to proceed with a national FP registry without cancer specialist involvement in the registry. More than half of the fertility services surveyed indicated that they would complete the web-based Australasian Oncofertility Registry (AOFR) during a patient consultation and approximately $27 \%$ reported that they would complete the webbased registry (AOFR) after the consultation[51]

In 2012 Klonoff-Cohen published details of two feasibility studies about establishing a fertility preservation database in the USA. ${ }^{[2]}$ The first study showed it was feasible to collect retrospective fertility preservation data from the 11 Society for Assisted Reproductive Technology (SART) affiliated fertility centres but the study highlighted the limitation with collecting cancer data without the collaboration between cancer and fertility centres and emphasized the limitations of retrospective oncofertility data collection. The second feasibility study documented that adequate patient numbers could be recruited by replicating the FP 
pilot study in all the SART centres, therefore providing enough patient data to provide evidence on uptake, utilisation and feasibility of FP from a medical, ethical and legal perspective in the USA.

Both the Australian and US studies have highlighted important feasibility issues for consideration when developing a complete population-based fertility preservation registry and these recommendations have been incorporated into the planning and development of the AOFR (cost, confidentiality, time taken for data entry, collaboration and quality assurance).

\section{The Australasian Oncofertility Registry}

The Australasian Oncofertility Registry (AOFR) is an innovative research study that will offer detailed information about each patient with regards to his/her demographic details, type of cancer diagnosis and cancer and fertility treatment in patients of reproductive age.

The AOFR will highlight short and long term reproductive function of cancer patients after cancer treatment by cancer type, treatment and age at diagnosis by collecting prospective fertility follow up data for all patients on the AOFR. Over time the registry will collect data on uptake and use of ART post cancer treatment (in patients who have or have not had FP at diagnosis), and data on successful pregnancy outcomes either with the use of ART or via natural conception.

The AOFR will allow us to monitor referral pathways nationally in two countries from cancer to reproductive specialist as well as the uptake of oncofertility services over time. Evidencebased research outcomes will be available to provide data for clinicians and patients as well as providing data to be able to advocate for improved oncofertility care, service planning and fostering national research programs. 


\section{Aims of the Australasian Oncofertility Registry}

The Australasian Oncofertility Registry collects oncofertility data from cancer and fertility centres in Australia and New Zealand on the patient's oncofertility journey under a number of themes (see table 1).

\section{Hypothesis}

Accurate and comprehensive national reporting of oncofertility management can inform future best practice and be benchmarked against the Australasian Oncofertility Consortium Charter, ${ }^{[52]}$ as well as improving allocation of oncofertility resources and research.

\section{Methodology}

The AOFR is a prospective international (Australia and New Zealand) population-based online database that enables the collection of oncofertility data that will be used to describe and compare oncofertility care and outcomes within, and between, cancer and fertility centres, as well as providing details on the long-term reproductive health of cancer patients following successful cancer treatment.

An oncofertility data dictionary was initially developed by the members of the Fertility Society of Australia Medical Preservation of Fertility Special Interest Group' and adapted over a 2year period following extensive consultation with the chief investigator group, oncology and fertility specialists, clinical trial fertility and advocacy special interest groups. Forty-three consumers, consisting of AYA and adult cancer patients and parents of paediatric cancer patients were involved in the development of the FUTuRE Fertility research study protocol. This group was also instrumental in developing the Australasian Oncofertility Consortium Charter ${ }^{[52]}$ which will be used to benchmark change in oncofertility practice overtime. The oncofertility data dictionary has been converted into an online web-based registry, available on any computer with internet connection through the website login page www.futurefertility.com.au by the IT team of Salesforce.com inc who built the AOFR as part of the company's technology innovation philanthropic giving program. The research study group felt that an international platform would be the most appropriate to support the AOFR given 
that the research group will extend participation to international collaborators in the second phase of the research study. The data is stored in a health cloud in the USA and the registry has compliance with HIPPA and Truste policy which govern the security and confidentiality of data security as well as who is able to access confidential information.

Clinicians will be given access to view specific details aligned to patients attending cancer and fertility consultations at their specific site. Analysis of data and publication of research findings will be conducted using de-identified data. To minimise data entry error and the time burden for clinicians, drop-down boxes are provided for most categories. Alternatively, data can be scribed on a paper-based form, which will be entered manually onto the AOFR.

The study has a National Project Manager and fractional clinical research associates (CRA) who will be responsible for site specific states and territories. Their role will be to ensure that the governance policies that support the AOFR are followed, to assist and support clinicians with data entry, and to undertake file-based audits for data completion and audit accuracy of data entered.

The AOFR is based on an 'opt-in' consent model, whereby patients are given information outlining the nature and purpose of the study and are offered an opportunity to ask questions. Patients are provided with the option to request withdrawal of data. Study information can be discussed by any health care professional on the patient's treatment team but the study consent will be taken by a medical professional/researcher between day 0-90 from first or relapsed diagnosis. A current amended has been submitted to extend this to day 180 .

Patients who consent to participate on the AOFR are given a unique identifying number. The consent is then scanned and uploaded onto the AOFR. Staff from participating hospitals and/or fertility centres can enter data once a patient has consented to participate. 


\section{Data Collection}

Data from the AOFR will be collected at diagnosis, end of treatment and then annually once a patient reaches 16 years of age. The data is entered onto the AOFR within eight sections (detailed below). Each section requires less than 3 minutes to complete. The first four sections are required to be completed by the cancer specialist, and sections five and six will be completed by the fertility specialist (reproductive endocrinologist, Andrologist, paediatric gynaecologist or paediatric endocrinologist). The remaining two sections will be completed by the specialist following the patient. It is intended that data will continue to be collected from patients over a 20-year period in order to collect longitudinal data on natural fertility outcomes and the use and success of ART following FP. This will provide robust data for paediatric and adolescent and young adult patients.

Section 1 - Demographic characteristics - this section has been designed to examine the demographic characteristics of patients and to elicit how uptake and referral for FP may differ depending on socioeconomic status as well as cultural and religious factors. All the data is presented in eight-digit format as this will provide details as to the timing of referral, consultations and FP procedures. Details on the patient's cancer centre and fertility centre postcode will enable geo-mapping to FP services (see table 2).

Section 2- Cancer diagnosis- in phase 1 of the registry, only patients with a malignant cancer diagnosis or who have a bone marrow transplant for non -malignant indications will be included. The diagnosis is recorded with the use of dropdown boxes, which have listed the cancer category, cancer subgroup, diagnosis and stage (see table 3).

The initial drop down box has three cancer categories - oncology, haematology malignant and BMT non-malignant. If oncology is selected, there are 15 subcategories available for selection (brain tumours, breast tumours, carcinoma, endocrine tumours, ENT tumours, upper Gl tumours, lower Gl tumours, germ cell tumours, gynaecological tumours, lung tumours, neuroblastoma family tumours, neuroendocrine tumours, sarcoma tumours, skin tumours, urogenital tumours). If haematology is selected, three subcategories would be available to 
choose (lymphoid neoplasms, myeloid neoplasms and lymphoma) and if non -malignant BMT is selected, then three subcategories are available to choose from (immunodeficiences, bone marrow failure and metabolic disease). In each of these subcategories, the clinician can select the diagnosis.

Section 3 - Cancer treatment- the FUTuRE Fertility research study team have summarised cumulative dose of paediatric and adult chemotherapy and BMT treatment protocols currently used in Australia and New Zealand. This will allow clinicians to identify the treatment protocol limiting the inaccuracy in data entry regarding treatment, and ensuring that clinicians will be able to enter data in a timely fashion. If patients are being treated on a new or modified protocol, not available on the registry, the clinician can upload the protocol for the research team. The AOFR will document the dose and field in sites that cover gonadal tissue and the neuroendocrine axis.

Section 4- Cancer specialist referral - this section has been designed to document communication about FP and patient barriers surrounding FP consultation and pursuit of FP strategies. The AOFR will also capture patients who are not referred by a cancer specialist but self-refer or are referred via primary health care provider (see table 4).

Section 5 - Sexual and reproductive health - this section will collect information on sexual and reproductive health factors which may affect the fertility potential and the choices that clinicians and patients make (see table 5).

Section 6- Fertility preservation strategy- the registry has two separate pages for male and female FP strategies allowing the research team to collect information on FP consultations, procedures undertaken, quality of gonadal tissue/gametes/embryos collected, and complications as a result of FP techniques (see table 6 and 7).

Section 7 - Follow Up- for patients over 16 years, clinical and laboratory reproductive health data which may be collected during the patient's routine clinical follow-up and will be reported 
to the register annually. The will include any tests regarding reproductive potential after treatment and the timing of return of normal fertility function (blood hormone tests, ultrasound scans in female patient's semen analysis in male patients). Patients younger than 16 years of age will only have confirmation of health status collected (see table 8).

Section 8- Starting a family after cancer- includes data collected on patients who have either had natural pregnancies or who have pursued ART, surrogacy or adoption to have children. This section documents the choices patients and clinicians make, pregnancy success rates and information about the patient's pregnancy and birth outcomes (see table 9).

\section{Recruitment Centres}

Currently 174 cancer and fertility centres have agreed to participate. These centres represent the paediatric oncology cancer centres, youth cancer services and fertility centres as well as many of the adult cancer treating centres that have clinical practices for tumours commonly seen in reproductive age patients. Over the first year these sites will be added in groups once local education and training has been completed. It is anticipated that following publication of the first annual report, the remaining adult cancer centres will participate, ensuring that the AOFR will have the opportunity to report national population data. In order to ensure that we are collecting population information, the data will be linked with the cancer registry data and details completed on the non-participation form.

\section{Reporting Timeframe}

Data is being collected at diagnosis, relapse, end of treatment and then annually. An annual report will be published 3 months after the first complete year of data collection and participating centres will also receive aggregate local data with information benchmarked against bi-national aggregate data. A number of peer-reviewed papers will be published, which will summarise the results based on the aims of the AOFR. 


\section{Population and recruitment}

Phase 1 of the AOFR will collect data for new or relapsed patients at diagnosis.

\section{Registry patient inclusion criteria}

All AYA patients (13-25 years old) and adult patients (26-45 years old) diagnosed with a malignant cancer will be eligible to have data included on the AOFR irrespective of whether they have received a referral and presented for a FP consultation. This will allow us to collect population data on oncofertility care and referral practices as well as data on reproductive potential and pregnancy outcomes.

Paediatric patients diagnosed with a malignant cancer aged 0-12 years are eligible to have data included on the AOFR if they are referred for a FP consultation. This will allow us to document the change in oncofertility care for children. Currently paediatric FP requires stringent oversight and generally is not undertaken as routine clinical practice. ${ }^{[53]}$ In this setting, the role of the AOFR involves data collection, synthesis and reporting with a reliance on participating centres to develop their own protocols for institutional governance and oversight.

\section{Registry patient exclusion criteria}

In the first phase of the AOFR we will not include patients if they have had gonadotoxic treatment for a non-cancer indication with the exception of non-malignant BMT. The AOFR will not capture data on FP procedures that siblings or parents undertake on behalf of a cancer patient. Other exclusion criteria include: patients who do not consent to data being collected and reported; patients whose first language is not English when cancer and fertility centres are unable to provide an appropriate interpreter to consent a patient onto the study; patients older than 45 years of age and cancer patients aged 0-12 years of age who are not referred for FP.

\section{Consent}

Age-appropriate information will be provided for consent and assent to the AOFR. The AOFR 
consent form will also invite patients to consent to be contacted about further research studies. Australian patients will also be asked to consent to Medicare data being collected to undertake a health economics modeling study to determine the cost of providing a FP program for all cancer patients at diagnosis and the medical and psychological costs in relation to delaying FP until after completion of cancer treatment. Finally consent will be taken for data linkage to other datasets in order to provide a complete oncofertility picture (cancer registries, National Death Index, Australian and New Zealand Assisted Reproduction Database, Perinatal Data Collection, Admitted Patient Data Collection).

Consent protocols vary slightly between the states. Generally patients older than 18 years of age will be able to provide their own consent to the AOFR after they have received the 'Patient Information Sheet' about the registry. Younger patients will receive an age appropriate information sheet and those who have understanding of the study will be invited to provide assent. These patients younger than 18 years will generally require parental consent. Some mature minors may provide their own consent but this will be judged according to the circumstances and varies in the different states.

The study team will ensure that the parent has received the appropriate 'Parent Information Sheet'. If a patient or parent notifies the AOFR of withdrawal of consent, the data of that patient will not be collected from the date of withdrawal. Patients and parents who do not consent to participate in the AOFR will be asked for reasons regarding non-participation to allow for (if necessary) improvements to be incorporated into specific areas of the research study.

Young patients after their $18^{\text {th }}$ birthday will receive a letter from the FUTuRE Fertility research team summarising the research study that they have been involved with and the benefits of their contributions to the AOFR to date. The letter will also include a copy of the adult revocation form. An 'Opt Out' process for consenting patients after their 18th birthday has been adopted by the study in order to continue to maintain robust data collection on this study population. 


\section{Governance Structure of the AOFR}

The AOFR has a governance structure which meets the Australian Commission on Safety and Quality in Health Care (ACSQHC) Strategic, Operational and Technical Principles. ${ }^{[54]}$ The AOFR governance structure is comprised of four groups that work together to ensure that the AOFR runs efficiently, operates at all times within legal constraints, particularly with regard to data security and confidentiality; is able to monitor outcomes and deals appropriately with clinical issues arising from data analysis; is appropriately managed by people who have clearly identified roles and responsibilities; and has processes for engagement and commitment of all relevant stakeholders. The governance structure will be reviewed on an annual basis to ensure the demands of ACSQHC Strategic, Operational and Technical Principles are being adhered to. A summary of each group and their function is summarised below:

1. The FUTuRE Fertility Research Group is responsible for managing the day-to-day aspects of the AOFR. This group comprises the Data Custodian, the Chief Investigator, the National Project Manager and CRAs in each state. The FUTuRE Fertility Research Group will be responsible for local training and governance issues and adherence to the standard operating policies (AOFR Quality Assurance and Data Management Policy (Version 1.4, Dated 21st October 2014, AOFR Data Access Policy (Version 1.4, Dated 21st October 2014), AOFR Data Security Policy (Version 1.4, Dated 21st October 2014), AOFR Data Custodian Policy (Version 1.4, Dated 21st October 2014), AOFR Publication Policy (Version 1.4, Dated 21st October 2014), AOFR Intellectual Property Policy (Version 1.4, Dated 21st October 2014), AOFR Consumer and Community Participation Statement Policy (Version 1.4, Dated 21st October 2014), AOFR Governance Policy (Version 1.4, Dated 21st October 2014).

2. The AOFR Steering Committee oversees the governance structure of the AOFR and maintains confidence of all aspects of the study with all parties. It provides strategic direction and ensures that deliverables are met in a timely fashion. The AOFR Steering Committee 
consists of two consumer members all the Chief Investigators and Associate Investigators plus the National Project Manager and the Data Custodian.

3. The Investigation Group consists of one fertility and cancer specialist (paediatric and adult) from each state and territory in Australia and New Zealand, the lead clinician, the National Project Manager, and the Data Custodian. This group provides feedback about local recruitment and study management; provide local assistance to other investigators in each state and provides feedback to the steering committee about any governance issues.

4. The FUTuRE Fertility Consumer Group ensures that consumers are involved in the development of all research studies and interpretation of results. There will be two consumer members represented on this committee at any given time, and representation will be made up of members from different states.

\section{Privacy and Confidentiality}

The FUTuRE Fertility Research Team respects the confidentiality and privacy of all patients during consultations, the consent process, and in relation to collection of patient data in keeping with the Australasian Oncofertility Registry Data Security Policy (Version 1.4 dated 14th of October 2014) and the National Statement on Ethical Conduct in Clinical Quality Registries. ${ }^{[55]}$ The registry data will only be used as outlined in the consent form and information sheets.

\section{Data Security}

All data collected will reside on a professionally maintained and physically secure server hosted by Salesforce.com located at an external site in the United States of America. Individual user sessions will be uniquely identified with each transaction and user logins will not be accessible by Salesforce.com personnel. The hardware and software configurations of the Salesforce.com system have been designed to provide secure user data that permit each user to view only its centres data as per the AOFR Data Security Policy (Version 1.4, Dated 21st October 2014). In addition to Salesforce.com disaster recovery capabilities, user data is 
also backed in a separate data center. Data is not taken offsite from the data centre, reducing the risk of loss or breach in security.

All paper-based information will be stored in a locked filing cabinet. After the data has been entered manually, paper-based forms will be shredded prior to disposal in accordance with the hospital disposal of medical information policy and ethical protocols. Validation of paper based data forms occurs prior to disposal, in line with the AOFR Quality Assurance and Data Quality policy.

\section{Intellectual Property (IP)}

The University of New South Wales is the licensee for the AOFR and holds the licence on behalf of the parties to use AOFR material (including IP) for academic, research and teaching purposes, including publication in peer-reviewed journals, unless otherwise determined by existing funding agreements. Access and permission to publish is, however, granted by the AOFR Steering Committee (AOFR Intellectual Property Policy: Version 1.4, Dated 21st October 2014).

\section{Data Access}

The data access policy defines how data can be obtained from the AOFR. This policy includes the criteria, conditions, and limitations for data access, procedures to be followed when requesting data access and the associated costs for such access. The outlined procedures have been put in place to protect against potential breaches of privacy as well as to ensure appropriate ethical integrity and scientific merit of proposals using AOFR data. In considering the approval of access to AOFR data, the AOFR Steering Committee seeks to balance the protection of AOFR registrant privacy with that of the public health significance from the proposed research.

\section{Ethics}

The study was approved by the South Eastern Sydney Local Health District Human Research Ethics Committee, HREC reference HREC/14/POWH/471); ACT Health Human Research 
Ethics Committee ETH.11.14.322, Queensland Health Human Research Ethics Committee HREC/14/QRCH/406/AMO2, Tasmanian Human Ethics Committee HOO14609, South Australia Human Ethics Committee HREC-14-QRCH-406, Medicare MI2893, Genea GEC0019, Melbourne IVF HREC 29/13-MIVF, IVF Australia (110) and Monash IVF (Epworth Group) HREC 673-15, Queensland Fertility Group (QFG) and St Andrews HREC South Australia \#95. Ethics is pending approval by Western Australian Human Research Ethics Committee HREC 2015001 and Victoria. Individual site- specific agreements are in the process of being signed for the 174 centres participating.

\section{Discussion}

The current national lack of evidence-based oncofertility data highlights the critical need for the Australasian Oncofertility Registry (AOFR). Timely referral and uptake of FP is important, however commencement of early cancer treatment is also critical. The AOFR will provide evidence-based population data on national uptake, utilisation and success of various FP options. This data will be used to inform the ongoing development of oncofertility services and pathways in Australia and New Zealand and will be used to develop evidence based guidelines for FP referral, treatment and fertility related psychosocial support. This data will also assist in helping to define the future resource allocations needed to develop national oncofertility services and advocate for an implementation of oncofertility services within every cancer service.

It is important that the AOFR will be documenting data on the complications of FP so that oncofertility supportive care national guidelines can be developed as well as giving reassurance to clinicians that it is possible to carry out FP in a timely and safely manner.

Currently, there is still inequity in access to oncofertility services and fertility preservation procedures and follow- up, with limited resources available in some rural areas. ${ }^{[37,56,57]}$ There is also limited data indicating that infertile non-cancer patients from lower socioeconomic groups and some cultural groups have insufficient access to assisted reproductive treatment. ${ }^{[58-61]}$ To date, this data has not been collected from cancer patients having FP or 
ART. The AOFR will be able to document not only the patient and clinician barriers to oncofertility care but will be also able also to document any inequity in access for lower socioeconomic areas or for patients from minority immigrant and indigenous communities by geographically mapping referral and uptake of FP/ART based on social determinants and ethnicity.

Paediatric oncofertility is an emerging clinical practice that carries significant ethical and practical challenges. ${ }^{[62-64]}$ Paediatric FP should only be considered in centres with stringent clinical and ethical and research governance protocols in place and the AOFR will ensure that new paediatric oncofertility services will be developed in a safe, equitable and evidence based manner.

The available data on the risk of cancer treatment causing infertility has significant and wellrecognised limitations as it does not include a patient's age or consideration of a patient's precancer risk factors for infertility or the increased risk for those whose treatment includes multimodality cancer treatment. As targeted medicine becomes more available we urgently need data on new and novel agents. We are fortunate to have fertility risk tables for some of the common cancer types but the documented risks of infertility quoted are very vague with short follow up, and are not always helpful for providing clinicians or patients with data that will help make choices. ${ }^{[65]}$ The AOFR will be able to use prospective data to develop more accurate risk projections for all cancer treatments, and therefore develop tools to predict fertility risk for treatments based on age and type of treatment used.

The Children's Cancer Survivor Study published a large cohort study on self-reported infertility, infertility treatment and time for first pregnancy. ${ }^{[66]}$ From the 20,000 childhood cancer survivors aged under 21 years at diagnosis and a survivor of $>5$ years included in the cohort (1970 to 1986) 3531 patients aged 18-39 years at the time of the study were eligible and consented to completing a retrospective questionnaire. This study showed that cancer survivors had a higher total risk of infertility compared to sibling controls ( $16 \%$ vs $11 \%$ ) with a relative risk of 1.48 (95\% Cl 1.23-1.78 and experienced more clinical infertility (13\% vs $10 \%)$ 
than sibling controls. The relative risk of infertility in survivors was significantly higher in those patients aged $<24$ years compared to those aged 30-40 years of age. The paper highlighted that, survivors were half as likely to be treated for infertility (44\% vs $75 \%$ ) than siblings.

Clearly, in-depth analysis is required. The AOFR will prospectively follow patients when they are wanting to conceive, and will report on the success on both natural and assisted reproductive therapy while also looking at other choices that cancer patients make, such as adoption. This data will be used by health care professionals to give patients evidence-based information on the use and success of ART in cancer patients. ${ }^{[66]}$

Finally, the engagement with and support for this research project to date from cancer and fertility health care professionals, advocacy groups and patients demonstrates how important this project is to both patients and clinicians.

\section{List of abbreviations}

1. AOFR - Australasian Oncofertility Registry

2. ART - Assisted Reproductive Treatment

3. AYA - Adolescent and Young Adult (15-25 year olds)

4. CRA - Clinical Research Assistant

5. FP - Fertility Preservation

6. FPS - Fertility Preservation Services

7. Fertility Specialist -reproductive endocrinologist, Andrologist, paediatric gynaecologist or paediatric endocrinologist.

8. ISFP - The International Society of Fertility Preservation

9. SART - Society for Assisted Reproductive Technology

10. TSE - Testicular sperm extraction

\section{Competing interests}

The authors declare that there are no conflicts of interest.

AA is funded by the Kids Cancer Alliance and CanTeen Australia.

YJ is supported by an Early Seed Career Grant from the Victoria Cancer Agency. 
CJS oversees a clinical FPS with a research focus and the program has received nondirected grants from MSD and Merck Serono to assist with database creation and FPS laboratory research. CJS has not received any funding for her involvement, nor any travel or educational grants. CW is supported by a Career Development Fellowship from the National Health and Medical Research Council of Australia (APP1067501) and an Early Career Development fellowship from the Cancer Institute of NSW (ID: 11/ECF/3-43). The Behavioural Sciences Unit is supported by the Kids with Cancer Foundation.

\section{Authors' contributions}

All authors contributed to the design of the study, the drafting of the study protocol and have approved the final manuscript.

\section{Authors information}

1. AA qualifications BSc. MBBS MSc Adolescent Certification in AYA Oncology

Director of the Sydney Youth Cancer Service at Sydney Children's Hospital and Prince of Wales Hospital

AA is a paediatric and adolescent oncologist whose main area of interest is in adolescent and young adult cancer, oncofertility, sexual health and sexual dysfunction in cancer patients.

2. CS qualifications MBBS FRANZCOG, FRCOG CREI

Associate Professor of $\mathrm{O}$ and G, University of Melbourne, Royal Women's Hospital, Melbourne. Head, Endocrine and Metabolic Service, Royal women's Hospital, Clinical Director, Melbourne IVF, head of FPS RWH and MIVF, head of Clinical research MIVF, Chair COSA FPS group

3. RM qualifications FRACP, PhD. Endocrinologist and NHMRC Research Fellow at The Hudson Institute, Monash Medical Centre Clayton, VIC. Consultant andrologist to the Monash IVF program and Director of Andrology Australia with a special interest in male fertility and the education of the community and profession male FP

4. BG qualifications BSoc.Sci MPh

National Project Manager, FUTuRE Fertility Study, Sydney Children's Hospital 
$B G$ is an epidemiologist and statistician whose main areas of interest include international women's and children's health, reproductive medicine, sexual health and paediatric oncology. 5. FA qualifications BSc (Hons), GradDipEd(Sec), Grad.Cert.Mgt FA is the Clinical Research Manager at Melbourne IVF. FA co-ordinates the fertility preservation program at Melbourne IVF. She is also the secretary of the Medical Preservation of Fertility Special Interest Group at the FSA

6. RJC qualifications: MB BCh (Rand), DCH (SA), FCP (SA), FRACP

Head, Clinical Oncology and Director of the Survivorship Program, Kids Cancer Centre, Sydney Children's Hospital, and Co-Director (Paediatric), NSW Cancer Survivor Centre, UNSW, Sydney, Australia

7. YJ qualifications MBBS, FRANZCOG, PhD. Paediatric and Adolescent Gynaecologist Royal Children's Hospital Melbourne, Lead investigator Fertility Preservation Taskforce Royal Children's Hospital, Gynaecologist Oncology Dysplasia Unit and Endocrine Metabolic Service Royal Women's Hospital, Senior Lecturer University of Melbourne. Main areas of interest are young women's health, governance of FP in children and adolescents, human papillomavirus. 8. CW qualifications BPsych (hons 1), MPH, PhD. Program leader, Behavioural Sciences Unit proudly supported by the Kids with Cancer Foundation, Kids Cancer Centre, Sydney Children's Hospital, Randwick, NSW, Australia; Senior research fellow, School of Women's and Children's Health, UNSW MEDICINE, University of NSW, Sydney, Australia; Research development fellow, Cancer Institute of NSW, Australia. Main areas of interest are psychooncology, mental health, paediatric cancer and health services research.

9. GD qualifications BPharm, MHA, Senior Pharmacist Paediatric Oncology Haematology at Sydney Children's Hospital and Prince of Wales Hospital.

GD is a paediatric and adolescent oncologist pharmacist main area of interest in medication safety.

10. DC qualifications BPharm MSHP

Clinical Pharmacist, Prince of Wales Hospital

$\mathrm{DC}$ is a clinical pharmacist specializing in adult oncology and haematology.

11. LG qualifications BPharm.

Paediatric Oncology and Haematology Clinical Pharmacist, Sydney Children's Hospital 
LG is a Clinical Pharmacist whose main areas of interest include paediatric oncology

12. MK qualifications BSc Hon MBBS

Project Manager, Fertility Preservation Taskforce, Royal Children's Hospital and Department of Obstetrics and Gynaecology, The University of Melbourne. Main areas of interest include the ethics and governance of Fertility Preservation in children and adolescents.

13. LMO qualifications MBBS FRACP. Medical Director ONTrac at Peter Mac, Victorian Adolescent and Young Adult Cancer Service; Paediatric and Adolescent Oncologist Childrens Cancer Centre, Royal Children's Hospital Melbourne. Main interests are adolescent and young adult cancer, sarcoma, fertility preservation and reproductive health, and cancer survivorship.

14. HW qualifications MS,MA,PhD. HW is senior statistician in the Kirby Institute with an interest in reproductive health and HIV acquisition.

15. RV qualifications M. Ec. PhD . RV, Director and Professor of Health Economics, Centre for Health Economics Research and Evaluation, UTS Business School, University of Technology Sydney

16. LG qualifications - BA (Hons) MA (Oxon) PhD. LG is a Clinical Ethics expert.

17. RD qualifications MBBS MMed FRANCOG CREI. RD is a lecturer at UNSW and a gynecologist at $\mathrm{RHW}$ and $\mathrm{SCH}$.

18. MJ qualifications MBA B.Eng. Regional Vice President at salesforce.com. MJ in his current role manages the customer success group sales organization for the Asia Pacific region. In his current role, he helps customers be successful by taking their concept/needs to deliver a technology solution.

19. JV qualifications MBA, B. Nursing. Senior Solution Architect at Salesforce. John Vu has over 15 years of consulting experience. He leads implementation team in his current role as a Senior Solution Architect delivering innovative business solutions from concept to production. 20. MC qualifications MBBS FRANZCOG FRCOG MD CREI MC is Professor of O\&G at UNSW. He is an infertility specialist dealing with oncology cases primarily referred from St George Hospital Kogarah. He is Chairman NPSEU Management Committee, Vice President Fertility Society of Australia, Vice Chair RANZCOG subspeciality Committee for Reproductive Endocrinology and Infertility 
21. WL qualifications MA, DPhil, FRCOG, FRANZCOG, CREI

Head of School of Women's and Children's Health, University of New South Wales, Director of Reproductive Medicine, Royal Hospital for Women, Senior Specialist, IVF Australia.

WL is an expert in infertility and reproductive medicine with a long standing interest in oncofertility and health economics of provision of care to couples with fertility problems.

22. EAS qualifications MBBS, MPH, MMED, MD, FAFPHM. Professor Public Health, University of Technology Sydney. EAS is a perinatal and reproductive epidemiologist whose main area of interest is in perinatal and reproductive surveillance systems and maternal and perinatal morbidity and mortality.

\section{Acknowledgements}

All the authors of the study gratefully acknowledge funding for this study provided by Salesforce, The Kids Cancer Centre Sydney Children's Hospital, CanTeen Australia, Merck Serono,Sir Asher Joel Foundation, Fertility Society of Australia and the Victorian Cancer Agency.

We also acknowledge the support given to our study by the patients, parents and partners who have taken part in the consultation, development and review of this study as well as members of the Medical Preservation of Fertility Special Interest Group within the Fertility Society of Australia.

We also acknowledge the support of the investigators in each participating cancer and fertility centre as well as the lead investigators in each state and territory that have provided invaluable assistance Dr Marianne Philips, Dr Rodger Hart, Dr Rachel Hughes, Dr Wayne Nicholls, A/Prof Anush Yazdani, Dr Ben Kroon, Dr Helen Irving, Dr Rick Walker, Prof Bogda Koczwara, Dr Fiona Young, Dr Michael Osborn, Dr Matthew Greenwood, Dr Tristan Petit, Dr Sarah Hunter and Ms. Meredith Woodhouse.

We also acknowledge the support provided by a number of cancer and fertility clinical trial and special interest and advocacy groups ensuring that the study not only aims to meet 
patient and clinicians needs but changes care for reproductive age patients with different cancer diagnosis- The Australian and New Zealand Children's Haematology and Oncology Group (ANZCHOG), Australian Sarcoma Study Group (ASSG), Australian Leukemia and Lymphoma Group (ALLG), The Australia and New Zealand Breast Cancer Trials Group (ANZBCTG), The Australia New Zealand Melanoma Trials Group (ANZMTG), Society of Adolescent and Young Adult Oncology (SAYA0), Andrology Australia, The Fertility Society of Australia (FSA) and CanTeen Australia.

AA is funded by the Kids Cancer Alliance and CanTeen Australia.

YJ is supported by an Early Seed Career Grant from the Victoria Cancer Agency.

CJS oversees a clinical FPS with a research focus and the program has received nondirected grants from MSD and Merck Serono to assist with database creation and FPS laboratory research. CJS has not received any funding for her involvement, nor any travel or educational grants. CW is supported by a Career Development Fellowship from the National Health and Medical Research Council of Australia (APP1067501) and an Early Career Development fellowship from the Cancer Institute of NSW (ID: 11/ECF/3-43). The Behavioural Sciences Unit is supported by the Kids with Cancer Foundation.

\section{Endnotes - none}




\section{References}

1. Australian Institute of Health Welfare (2012). Cancer in Australia An overview 2012. Cat no. 56. AlHW 2012.

2. Matthews ML, Hurst BS, Marshburn PB, Usadi RS, Papadakis MA, Sarantou T: Cancer, fertility preservation, and future pregnancy: a comprehensive review. Obstetrics and gynecology international 2012, 2012.

3. Zebrack BJ, Casillas J, Nohr L, Adams H, Zeltzer LK: Fertility issues for young adult survivors of childhood cancer. Psychooncology 2004, 13(10):689-699.

4. Rosen A, Rodriguez-Wallberg KA, Rosenzweig L: Psychosocial distress in young cancer survivors. Semin Oncol Nurs 2009, 25(4):268-277.

5. Klock SC, Zhang JX, Kazer RR: Fertility preservation for female cancer patients: early clinical experience. Fertil Steril 2010, 94(1):149-155.

6. Wallace WH, Thomson AB, Saran F, Kelsey TW: Predicting age of ovarian failure after radiation to a field that includes the ovaries. Int $J$ Radiat Oncol Biol Phys 2005, 62(3):738-744.

7. Sklar CA, Mertens AC, Mitby P, Whitton J, Stovall M, Kasper C, Mulder J, Green D, Nicholson HS, Yasui $Y$ et al: Premature menopause in survivors of childhood cancer: a report from the childhood cancer survivor study. J Natl Cancer Inst 2006, 98(13):890-896.

8. Wallace WH, Anderson RA, Irvine DS: Fertility preservation for young patients with cancer: who is at risk and what can be offered? The lancet oncology 2005, 6(4):209-218.

9. Levine J, Stern CJ: Fertility preservation in adolescents and young adults with cancer. Journal of clinical oncology 2010, 28(32):4831-4841.

10. Oeffinger KC, Mertens AC, Sklar CA, Kawashima T, Hudson MM, Meadows AT, Friedman DL, Marina N, Hobbie W, Kadan-Lottick NS et al: Chronic health conditions in adult survivors of childhood cancer. N Engl J Med 2006, 355(15):1572-1582.

11. Green DM, Sklar CA, Boice JD, Jr., Mulvihill JJ, Whitton JA, Stovall M, Yasui Y: Ovarian failure and reproductive outcomes after childhood cancer treatment: results from the Childhood Cancer Survivor Study. J Clin Oncol 2009, 27(14):2374-2381.

12. Gnaneswaran S, Deans R, Cohn RJ: Reproductive late effects in female survivors of childhood cancer. Obstetrics and gynecology international 2012, 2012:564794.

13. Wallace $\mathrm{WH}$, Anderson RA, Irvine DS: Fertility preservation for young patients with cancer: who is at risk and what can be offered? Lancet Oncol 2005, 6(4):209-218

14. von Wolff M, Montag M, Dittrich R, Denschlag D, Nawroth F, Lawrenz B: Fertility preservation in women--a practical guide to preservation techniques and therapeutic strategies in breast cancer, Hodgkin's lymphoma and borderline ovarian tumours by the fertility preservation network FertiPROTEKT. Arch Gynecol Obstet 2011, 284(2):427-435.

15. Cruz MRS, Prestes JÝC, Gimenes DL, Fanelli MF: Fertility preservation in women with breast cancer undergoing adjuvant chemotherapy: a systematic review. Fertility and sterility 2010, 94(1):138-143.

16. Kim SS: Fertility preservation in female cancer patients: current developments and future directions. Fertility and sterility 2006, 85(1):1-11.

17. Kondapalli LA: Oncofertility: a new medical discipline and the emerging scholar. In: Oncofertility Fertility Preservation for Cancer Survivors. edn.: Springer; 2007: 221234.

18. Zebrack BJ, Casillas J, Nohr L, Adams H, Zeltzer LK: Fertility issues for young adult survivors of childhood cancer. Psychooncology 2004, 13(10):689-699.

19. Rosen A, Rodriguez-Wallberg KA, Rosenzweig L: Psychosocial distress in young cancer survivors. Semin Oncol Nurs 2009, 25(4):268-277.

20. Loscalzo MJ, Clark KL (eds.): The Psychosocial Context of Cancer-Related Infertility. USA: T.K. Woodruff and K.A. Snyder (eds.) Oncofertility; 2007. 
21. Gorman JR, Bailey S, Pierce JP, Su HI: How do you feel about fertility and parenthood? The voices of young female cancer survivors. Journal of cancer survivorship : research and practice 2012, 6(2):200-209.

22. Oeffinger KC, Mertens AC, Sklar CA, Kawashima T, Hudson MM, Meadows AT, Friedman DL, Marina N, Hobbie W, Kadan-Lottick NS et al: Chronic Health Conditions in Adult Survivors of Childhood Cancer. N Engl J Med 2006, 355(15):1572-1582.

23. Redig AJ, Brannigan R, Stryker SJ, Woodruff TK, Jeruss JS: Incorporating fertility preservation into the care of young oncology patients. Cancer 2011, 117(1):4-10.

24. Klonoff-Cohen $\mathrm{H}$ : Establishing a fertility preservation database: no time like the present. Expert Review of Obstetrics \& Gynecology 2012, 7(3):213-225.

25. Oktay K, Sonmezer M: Ovarian tissue banking for cancer patients Fertility preservation, not just ovarian cryopreservation. Human reproduction 2004, 19(3):477-480.

26. Oktay K: Successful human ovarian autotransplantation to the upper arm. Cancer 2005, 103(9):1982-1983; author reply 1983.

27. Gook DA, Edgar DH: Human oocyte cryopreservation. Hum Reprod Update 2007, 13(6):591-605.

28. Stern C, Conyers R, Orme LM, Barak S, Agresta F, Seymour J: Reproductive concerns of children and adolescents with cancer: challeges and potential solutions. Clinical Oncology in Adolescents and Young Adults 2013, 2013(3):63-78.

29. Feldschuh J, Brassel J, Durso N, Levine A: Successful sperm storage for 28 years. Fertility and sterility 2005, 84(4):1017-e1013.

30. Sharma V: Sperm storage for cancer patients in the UK: a review of current practice. Human reproduction 2011, 26(11):2935-2943.

31. McNeil JJ, Evans SM, Johnson NP, Cameron PA: Clinical-quality registries: their role in quality improvement. The Medical journal of Australia 2010, 192(5):244-245.

32. Berookhim BM, Mulhall JP: Outcomes of operative sperm retrieval strategies for fertility preservation among males scheduled to undergo cancer treatment. Fertil Steril 2014, 101(3):805-811.

33. Holoch $\mathrm{P}$, Wald M: Current options for preservation of fertility in the male. Fertil Steril 2011, 96(2):286-290.

34. Sonmezer M, Oktay K: Fertility preservation in female patients. Hum Reprod Update 2004, 10(3):251-266.

35. Jeruss JS, Woodruff TK: Preservation of fertility in patients with cancer. New England Journal of Medicine 2009, 360(9):902-911.

36. Maltaris T, Weigel M, Mueller A, Schmidt M, Seufert R, Fischl F, Koelbl H, Dittrich R: Cancer and fertility preservation: fertility preservation in breast cancer patients. Breast Cancer Res 2008, 10(2):206.

37. McQuillan SK., Malenfant D., Jayasinghe Y., Orme L., SR. G: Audit of Current Fertility Preservation Strategies used by Individual Pediatric Oncologists Throughout Australia and New Zealand Journal of Paediatric Oncology 2013, 1:112-118.

38. Schover LR: Patient attitudes toward fertility preservation. Pediatr Blood Cancer 2009, 53(2):281-284.

39. Quinn GP, Vadaparampil ST, Lee JH, Jacobsen PB, Bepler G, Lancaster J, Keefe $\mathrm{DL}$, Albrecht TL: Physician referral for fertility preservation in oncology patients: a national study of practice behaviors. J Clin Oncol 2009, 27(35):5952-5957.

40. Tingen C: Discussing oncofertility; the oncologists responsibility 25 Sptember 2009 www.hemoncotoday.com/article (accessed 21st April 2015). HemOnc Today 2009.

41. Forman EJ, Anders CK, Behera MA: A nationwide survey of oncologists regarding treatment-related infertility and fertility preservation in female cancer patients. Fertil Steril 2010, 94(5):1652-1656.

42. Quinn GP, Block RG, Clayman ML, Kelvin J, Arvey SR, Lee JH, Reinecke J, Sehovic I, Jacobsen PB, Reed D et al: If you did not document it, it did not happen: rates of documentation of discussion of infertility risk in adolescent and young adult oncology patients' medical records. J Oncol Pract 2015, 11(2):137-144.

43. Levine JM: Preserving Fertility in Children and Adolescents with Cancer. Children 2014, 1(2):166-185. 
44. International Society for Fertility Preservation: About ISFP (Accessed webpage 22 January 2015) http://www.isfp-fertility.org/.

45. http://oncofertility.northwestern.edu/first-registry.

46. Ezcurra D, Rangnow J, Craig M, Schertz J: The Human Oocyte Preservation Experience (HOPE) a phase IV, prospective, multicenter, observational oocyte cryopreservation registry. Reprod Biol Endocrinol 2009, 7:53.

47. Barri PN, Coroleu B, Devesa M: Trend of Fertility Preservation Strategies in Europe. . In: 2nd World Congress on Fertility Preservation Miami Beach, FL, USA 2011:1-74.

48. von Woff M, Lawrenz B: FertiPROTEKT Network for Fertility Preservation Techniques before Chemo- AND Radiotherapy. Journal of Reproductive Medicine and Endocrinology 2013, 10(Special Issue 1):60-65.

49. Society for Assisted Reproductive Technology, [http://www.sart.org, (date Assesed 30 April 2015).

50. Macaldowie A., Wang YA., Chughtai AA., Chambers GM: Assisted reproductive technology in Australia and New Zealand 2012. In.: Sydney, National Perinatal Epidemiology and Statistics Unit, the University of New South Wales; 2014.

51. Anazodo A, Stern C, Agresta F, Chapman M, Sullivan E: Provision of Fertility Preservation Services in Australia and New Zealand. Submitted for publication.

52. Anazodo AC, Gerstl B, Stern C, McLachlan RI, Agresta F, Jayasinghe Y, Cohn R, Wakefield CE, Chapman M, Ledger W et al: Utilising the experience of consumers in consultation to develop the Australasian Oncofertility Consortium Charter Accepted for Journal of Adolescent and Young Adult Oncology (dated: 6th November 2015).

53. Loren AW, Mangu PB, Beck LN, Brennan L, Magdalinski AJ, Partridge AH, Quinn G, Wallace WH, Oktay K: Fertility preservation for patients with cancer: American Society of Clinical Oncology clinical practice guideline update. Journal of clinical oncology : official journal of the American Society of Clinical Oncology 2013, 31(19):2500-2510.

54. Barraclough $\mathrm{B}$, Birch $\mathrm{J}$ : Health care safety and quality: where have we been and where are we going? MJA 2006, 184(10):S48-S50.

55. http://www.nhmrc.gov.au/publications/humans/part1.htm. (Accessed 24 July 2009).

56. Oosterhuis BE, Goodwin T, Kiernan M, Hudson MM, Dahl GV: Concerns about infertility risks among pediatric oncology patients and their parents. Pediatr Blood Cancer 2008, 50(1):85-89.

57. Burns KC, Boudreau C, Panepinto JA: Attitudes regarding fertility preservation in female adolescent cancer patients. J Pediatr Hematol Oncol 2006, 28(6):350-354.

58. Fujimoto VY, Luke B, Brown MB, Jain T, Armstrong A, Grainger DA, Hornstein MD: Racial and ethnic disparities in assisted reproductive technology outcomes in the United States. Fertil Steril 2010, 93(2):382-390.

59. Seifer DB, Frazier LM, Grainger DA: Disparity in assisted reproductive technologies outcomes in black women compared with white women. Fertil Steril 2008, 90(5):1701-1710.

60. Jain $\mathrm{T}$ : Socioeconomic and racial disparities among infertility patients seeking care. Fertil Steril 2006, 85(4):876-881.

61. Chambers GM, Hoang VP, Illingworth PJ: Socioeconomic disparities in access to ART treatment and the differential impact of a policy that increased consumer costs. Human reproduction (Oxford, England) 2013, 28(11):3111-3117.

62. Fertility preservation and reproduction in cancer patients. Fertil Steril 2005, 83(6):1622-1628.

63. Quinn GP, Murphy D, Knapp C, Stearsman DK, Bradley-Klug KL, Sawczyn K, Clayman ML: Who decides? Decision making and fertility preservation in teens with cancer: a review of the literature. J Adolesc Health 2011, 49(4):337-346.

64. Stegmann BJ: Unique ethical and legal implications of fertility preservation research in the pediatric population. Fertil Steril 2010, 93(4):1037-1039.

65. Loscalzo MJ, Clark KL: The psychosocial context of cancer-related infertility. In: Oncofertility fertility preservation for cancer survivors. edn.: Springer; 2007: 180-190.

66. Barton SE, Najita JS, Ginsburg ES, Leisenring WM, Stovall M, Weathers RE, Sklar $\mathrm{CA}$, Robison LL, Diller L: Infertility, infertility treatment, and achievement of 
pregnancy in female survivors of childhood cancer: a report from the Childhood Cancer Survivor Study cohort. Lancet Oncol 2013, 14(9):873-881. 
Tables

Table 1 - Themes And Aims Of The Australasian Oncofertility Registry

\begin{tabular}{|c|c|c|}
\hline Number of Each Theme & Title of Each Theme & Aims Of Each Theme \\
\hline $\begin{array}{l}\text { Theme } 1 \text { and 2- Uptake and } \\
\text { utilisation of fertility } \\
\text { preservation }\end{array}$ & Referral and uptake of FP & $\begin{array}{l}\text { 1. To monitor the frequency } \\
\text { of referral and uptake of FP } \\
\text { in children, AYA and adults } \\
\text { with cancer aged } 0-45 \text { years } \\
\text { by age, cancer diagnosis and } \\
\text { treatment. } \\
\text { 2. To investigate the barriers } \\
\text { to FP by age, demographics, } \\
\text { cancer diagnosis and } \\
\text { treatment } \\
\text { 3. To investigate the } \\
\text { complications of FP, by age } \\
\text { of patient, cancer diagnosis } \\
\text { and FP strategy offered. }\end{array}$ \\
\hline $\begin{array}{l}\text { Theme 3- FP potential } \\
\text { following cancer treatment }\end{array}$ & $\begin{array}{l}\text { Fertility potential following } \\
\text { cancer treatment }\end{array}$ & $\begin{array}{l}\text { 1. To investigate and monitor } \\
\text { the return of reproductive } \\
\text { function following cancer } \\
\text { treatment. } \\
\text { 2. To determine the risk for } \\
\text { infertility by diagnosis and } \\
\text { cancer treatment type } \\
\text { following cancer therapy. } \\
3 \text { To characterise the long } \\
\text { term reproductive endocrine } \\
\text { function for cancer patients } \\
\text { after treatment }\end{array}$ \\
\hline
\end{tabular}




\begin{tabular}{|l|l|l|}
\hline Theme 4- ART following & $\begin{array}{l}\text { Assisted reproductive } \\
\text { treatment (ART) following } \\
\text { cancer treatment }\end{array}$ & $\begin{array}{l}\text { 1.To monitor the uptake of } \\
\text { ART by cancer patients post } \\
\text { cancer treatment } \\
\text { documenting ART choices, } \\
\text { complications and } \\
\text { pregnancies }\end{array}$ \\
& $\begin{array}{l}\text { 2.To monitor the rates for } \\
\text { natural and ART pregnancies } \\
\text { and births in cancer survivors }\end{array}$ \\
& & \\
\hline
\end{tabular}

\section{Table 2 - Demographics Data Dictionary For The Australasian Oncofertility Registry}

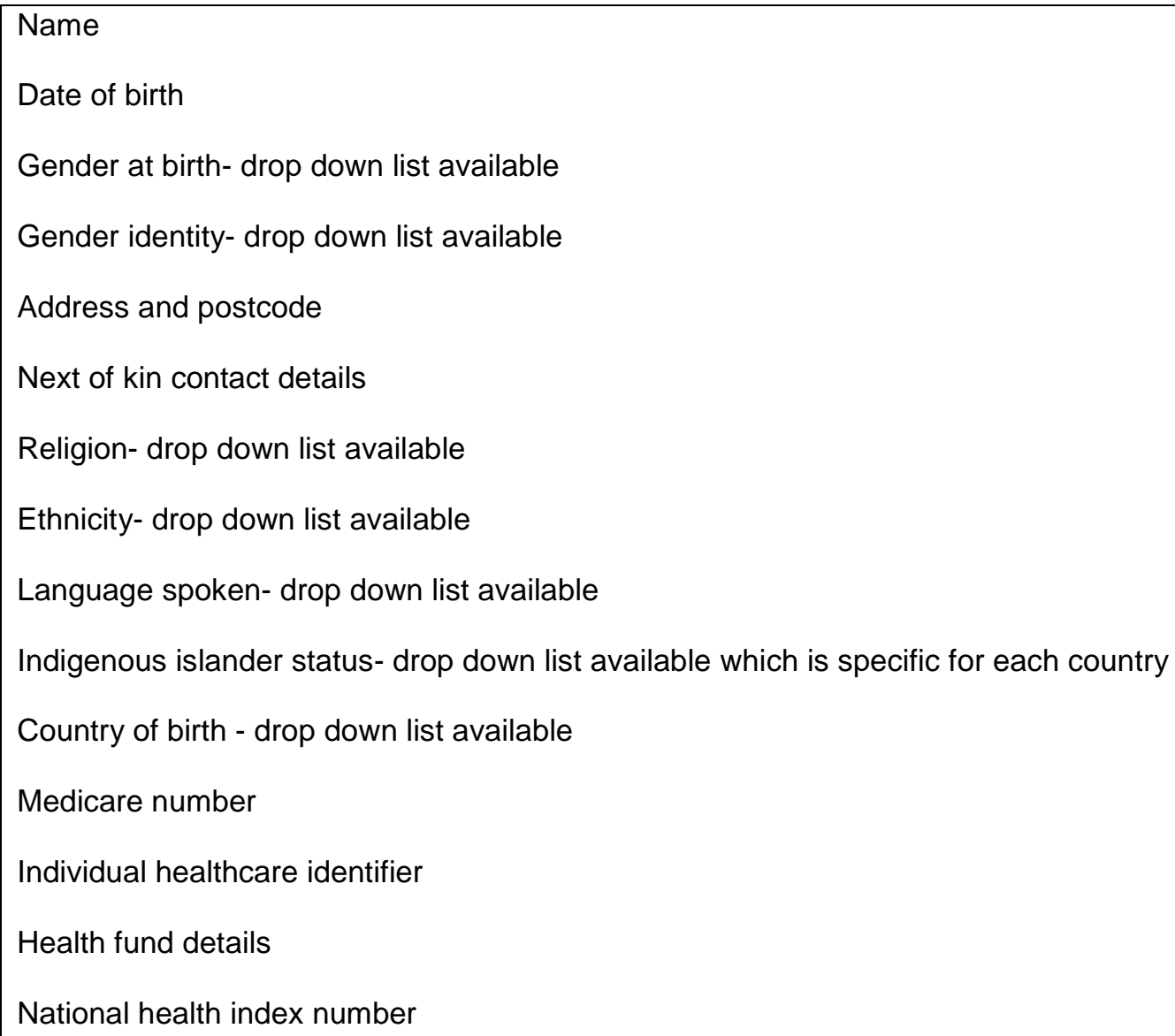


Table 3 - Cancer Diagnosis Data Dictionary For The Australasian Oncofertility Registry

Date of diagnosis

Date of death

Cancer centre postcode

Cancer diagnosis - new or relapse

Cancer category - oncology, haematology, bone marrow transplant (BMT) non malignantdrop down list available.

Cancer diagnosis and subgroup for each cancer category- drop down list available.

Table 4 - Cancer Specialist Referral Data Dictionary For The Australasian Oncofertility

\section{Registry}

FP information given to patient - yes/no

Type of FP information given - oral, verbal or both

Referred to FP services (fertility/andrology/reproductive endocrinologist, paediatric

gynaecologist or paediatric endocrinologist) yes/no

Referral date

Patient declined referral- yes $/$ no

Reasons for cancer clinician non- referral to FP services- drop down list available

Reason for patients declining FP specialist referral- drop down list available

\section{$\underline{\text { Table } 5 \text { - Sexual and Reproductive Health Data Dictionary For The Australasian }}$}

\section{Oncofertility Registry}

Sexually active prior to a cancer diagnosis- yes/no

Partner status- drop down list available

Sexuality- drop down list available 
Contraception use/type

For Male

Ability to achieve an erection - yes/no

Able to ejaculate - yes/no

Previous surgery to testes - yes/no

History of undescended testes- yes/no

\section{For Females}

Age of menarche

Menstruation prior to cancer diagnosis -yes/no

Regularity of menstrual cycle - less than 25 days, 26-35 days, greater than $36-50$ days, greater than 50 days

Number of pregnancies before cancer diagnosis - never pregnant, one pregnancy, two pregnancies, multiple pregnancies

Previous termination of pregnancy- yes/no

\section{Table 6 - FP Strategy (Female) Data Dictionary For The Australasian Oncofertility}

\section{Registry}

FP service postcode

FP consultation - yes/no

Date of consultation

Planned FP- yes/no

Type of FP- drop down list available

Use of gonadotrophin releasing hormone agonist (GnRH)- yes/no

Type of $\mathrm{GnRH}$ - drop down list available

Ovarian transposition - yes/no

Side- right, left, both 
Baseline bloods - drop down list available

\section{Oocyte and Embryo Cryopreservation}

No of oocytes collected

Number of mature oocytes frozen

No of embryos frozen at 2,3 and 5

\section{Ovarian Tissue Cryopreservation}

No of slices

Follicle density

Oocytes collected from tissue - yes/no

Primordial follicles seen- yes/no

Histology sent from ovarian samples- yes/no

Confirmed cancer - yes/no

\section{Complications}

Complications of $\mathrm{FP}-$ yes/no

Type of Complications- drop down list available

Table 7 - FP Strategy (Male) Data Dictionary For The Australasian Oncofertility Registry

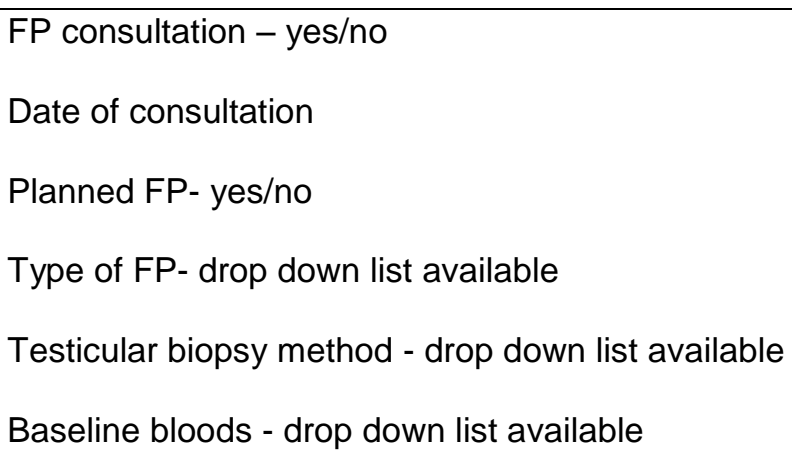




\section{Quality of Semen Collected}

Days of abstinence prior to procedure

Total sperm per ejaculate

Collection- vials or straws

Sperm morphology - normal forms \%

Concentration (million/MI)

Progressive motility (million/Ml)

\section{Complications}

Complications of FP treatment- yes/no

Complications-- drop down list available

Table 8 - Follow Up After Cancer Treatment Data Dictionary For The Australasian

\section{Oncofertility Registry}

\section{FEMALES}

Menstrual period after cancer treatment- drop down list available

Regularity of menstrual cycle- drop down list available

Last menstrual cycle- drop down list available

Hormone blood tests annually

\section{Pelvic Ultrasound}

Type of scan - transvaginal or transabdominal ultrasound

Endometrial thickness

Antral follicle count

Ovarian volume

Ovarian size in $3 D$

Number of follicles 2-6mm (antral follicle count)

Uterine size in 3D

Uterine volume 
Uterine pathology

\title{
BRAC mutation
}

BRAC1- not tested, yes/no

BRAC2 -not tested, yes/no

In phase 2 other predisposition genes will be added following consultation*

\section{MALES}

Hormone blood tests after cancer treatment

Sperm collection after cancer treatment

Days of abstinence prior to procedure

Collection- vials or straws

Sperm morphology - normal forms $\%$

Sperm concentration (million/Ml)

Total sperm per ejaculate

Progressive motility (million/Ml)

Post thaw motility\%

Number of straws/vials stored

Table 9 - Starting A Family After Cancer Data Dictionary (Items Recorded For Cancer

\section{Patient or Partner of Cancer Patient)}

\author{
Natural pregnancy - yes/no \\ Type of ART- drop down list available \\ Start date of ART \\ No of cycles \\ Pregnancy outcomes- drop down list available \\ Pregnancy complications < 14 weeks- drop down list available \\ Pregnancy complications $>14$ weeks- drop down list available \\ Date of birth of child
}


Sex of baby - male/female

Weight of baby

Gestation of baby- drop down list available 